\title{
Large Freshwater Phages with the Potential to Augment Aerobic Methane Oxidation
}

\author{
LIN-XING CHEN $^{1}$, RAPHAËL MÉHEUST ${ }^{1}$, ALEXANDER \\ CRITS-CHRISTOPH $^{2}$, KATHERINE D. MCMAHON ${ }^{3}$, TARA \\ COLENBRANDER NELSON $^{4}$, LESLEY A. WARREN ${ }^{4,5}$, AND \\ JILLIAN F. BANFIELD ${ }^{1,2,6,7}$
}

${ }^{1}$ Department of Earth and Planetary Sciences, Berkeley, CA, USA

${ }^{2}$ Department of Plant and Microbial Biology, University of California, Berkeley, Berkeley, CA, USA

${ }^{3}$ Departments of Civil and Environmental Engineering, and Bacteriology, University of Wisconsin, Madison, WI, USA

${ }^{4}$ Department of Civil and Mineral Engineering, University of Toronto, Toronto, Canada

${ }^{5}$ School of Geography and Earth Science, McMaster University, Hamilton, Canada

${ }^{6}$ Department of Environmental Science, Policy, and Management, Berkeley, CA, USA

${ }^{7}$ Earth and Environmental Sciences, Lawrence Berkeley National Laboratory, Berkeley, CA, USA

There is growing evidence that phages with unusually large genomes are common across various natural and human microbiomes, but little is known about their potential ecosystem impacts. Here, we reconstructed large phage genomes from freshwater lakes known to contain bacteria that oxidize methane. Twenty-two manually curated genomes with $159-527 \mathrm{kbp}$ in length were found to encode the pmoC gene, an enzymatically critical subunit of the particulate methane monooxygenase, the predominant methane oxidation catalyst in nature. The phage-associated PmoC show $>90 \%$ similarity and affiliate phylogenetically with those of coexisting bacterial methanotrophs, and their abundance patterns correlate with the abundances of these bacteria, supporting hostphage relationships. We suggest that phage PmoC has similar functions to additional copies of PmoC encoded in bacterial genomes, thus contribute to growth on methane. Transcriptomics data from one system showed that some phage-associated pmoC genes are actively expressed in situ. Augmentation of bacterial methane oxidation by pmoC-phages during infection could modulate the efflux of this powerful greenhouse gas into the environment. 